\title{
Method and key points for isolation of human amniotic epithelial cells with high yield, viability and purity
}

\author{
Hossein Motedayyen ${ }^{1}$, Nafiseh Esmaeil ${ }^{1}$, Nader Tajik², Fahimeh Khadem', Somayeh Ghotloo ${ }^{3}$, Behnaz Khani ${ }^{4}$ \\ and Abbas Rezaei ${ }^{1 *}$
}

\begin{abstract}
Objective: Human amniotic epithelial cells (hAECs) which are isolated from the amniotic membrane have stem cell-like properties and immunomodulatory effects. Several protocols have been proposed for isolation of hAECs, nevertheless, there is no report concerning isolation of highly viable hAECs, with desirable yield, and without significant purity reduction. In the current study, a detailed protocol with some modification of previous ones is presented in which the amendments led to isolation of hAECs with high purity, yield and viability. Moreover, isolated hAECs were subjected to immuno-phenotyping and their physiological status was assessed using a proliferation assay.

Results: The average yield of obtained hAECs using the new modified method was $190 \times 10^{6}$ cells with a mean viability of $87 \%$, with less than $1 \%$ contamination with mesenchymal stem cells (MSCs). The isolated cells were > $95 \%$ positive for the epithelial cell markers. The lowest initial plating efficiency of the cells was $80 \%$. Freshly isolated hAECs had the ability to proliferate for 5-6 passages in a standard culture medium.
\end{abstract}

Keywords: hAECs, Placenta, Amnion, Epithelial cells, Isolation

\section{Introduction}

Human placenta consists of three layers including amnion, chorion and deciduas [1]. The amnion layer, which is derived from the embryo, is the closest layer to the fetus and consists of both cubical and columnar epithelial cells [2-4]. These human amniotic epithelial cells (hAECs) have unique properties that distinguish them from other human cell types including immunomodulatory effects and stem cell-like features which provide capability to differentiate into different cell types originating from three germ layers without any of the ethical concerns related to human stem cells $[1,5-8]$. In addition, no tumorigenicity and transplant rejection were observed upon hAECs transplantation $[1,9]$. Therefore, hAECs could be safely employed in regenerative medicine and treatment of diseases with immune pathophysiology.
Until now, several protocols were reported by which hAECs were isolated with the average yield ranging from $8 \times 10^{6}$ to $120 \times 10^{6}$ cells and a viability of $83-98 \%$. The isolated cells were positive for the epithelial cell markers in range of $83-98 \%$, while contamination with MSCs was among $1-80 \%[3,10-15]$, nevertheless, as mentioned in Table 1, no report is available concerning isolation of highly viable hAECs with desirable yield and without any significant purity reduction. In this study, a detailed protocol with some modification of previous ones is presented in which by using the new modified method hAECs were isolated with high yield, viability and purity with the minimum contamination with other cell populations. In addition, the isolated hAECs were subjected to immuno-phenotyping, and their physiological status was assessed using a proliferation assay.

*Correspondence: Rezaei@mui.ac.ir; hm.k_65@yahoo.com

${ }^{1}$ Department of Immunology, Faculty of Medicine, Isfahan University

of Medical Sciences, Hezar-Jereeb Ave, Isfahan, Iran

Full list of author information is available at the end of the article 

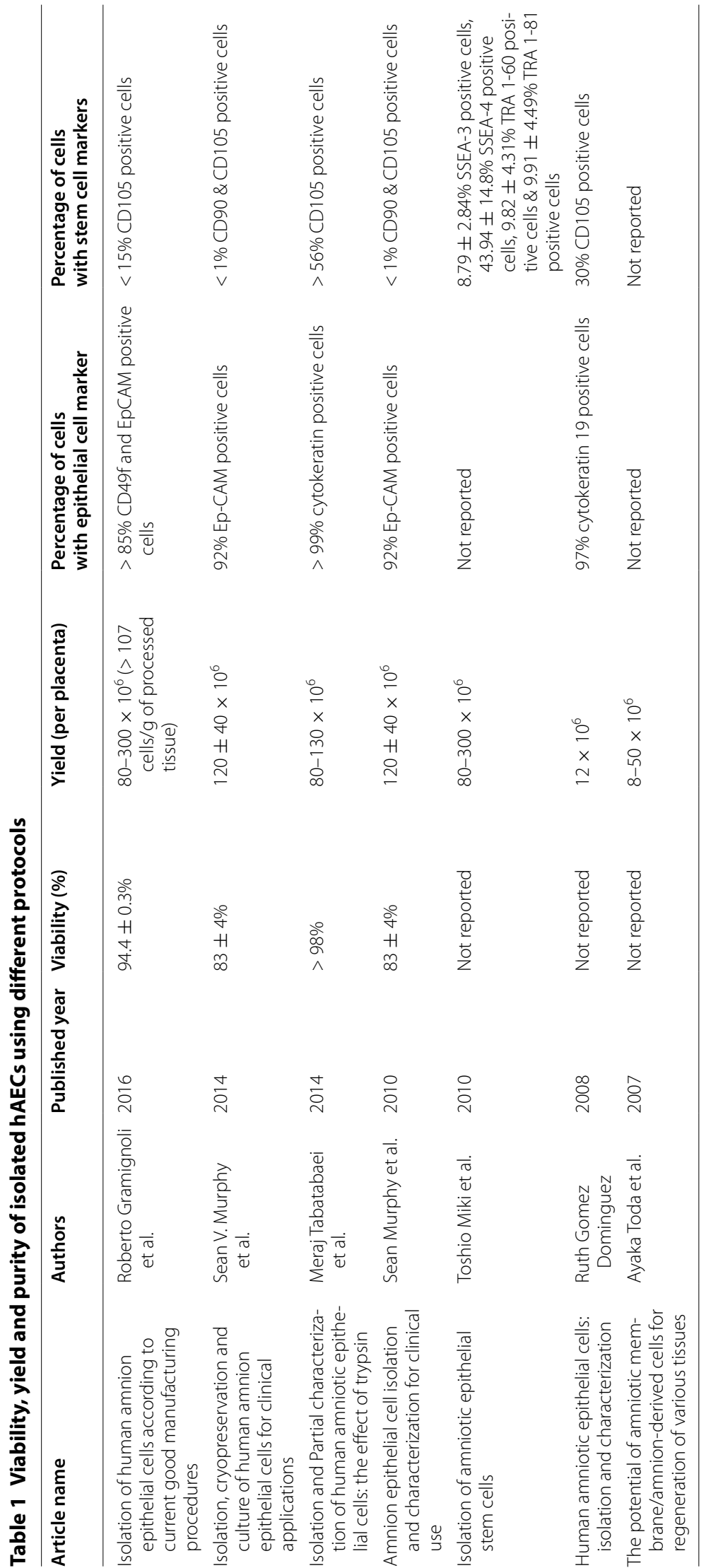


\section{Main text \\ Note}

All deliveries which were positive for infectious agents including HBV, HCV and HIV and those with pre-diagnosed genetic abnormalities were excluded from this study. Full-term human placentas were obtained from six healthy women with a normal singleton pregnancy undergoing uncomplicated elective cesarean section. All the materials and equipment used in this procedure were sterile.

\section{Methods}

\section{Preparation of reagents and solutions}

Standard culture medium DMEM/F12 (Gibco, Thermo Fisher Scientific, USA) supplemented with 10\% FBS, $100 \mathrm{U} / \mathrm{ml}$ penicillin, $100 \mu \mathrm{g} / \mathrm{ml}$ streptomycin (1X, Sigma Aldrich, USA) and $10 \mathrm{ng} / \mathrm{ml} \mathrm{EGF} \mathrm{(Thermo} \mathrm{Fisher} \mathrm{Scien-}$ tific, USA) was used as standard culture medium.

Pre-digestion buffer To prepare $1000 \mathrm{ml}$ of $1 \mathrm{X}$ Pre-digestion buffer, all the components mentioned in Table 2 were added to $900 \mathrm{ml}$ of tissue culture-grade water $\left(20-25{ }^{\circ} \mathrm{C}\right)$ and the $\mathrm{pH}$ was adjusted to 7.2 (It is because $\mathrm{pH}$ of the buffer rises up to $0.1-0.3$ units during filtration). Thereafter, tissue culture-grade water was added to the solution and the final volume adjusted to $1000 \mathrm{ml}$. The buffer was immediately sterilized by filtration using a $0.22 \mu \mathrm{m}$ filter (Thermo Fisher Scientific, USA) and stored at $4{ }^{\circ} \mathrm{C}$ for further usage.

\section{Isolation of hAECs from placenta \\ Isolation of the amniotic membrane}

The placenta was transferred to the laboratory in a sterile container which was filled with ice-cold Hanks' balanced salt solution (calcium- and magnesium-free HBSS) or RPMI/1640 (Gibco, Thermo Fisher Scientific, USA) containing $1 \%$ penicillin/streptomycin solution (Sigma Aldrich, USA) to cover up the placenta surface. The placenta was placed in a sterile container (under a horizontal laminar flow hood) while the amniotic membrane was

Table 2 Pre-digestion buffer ingredients

\begin{tabular}{ll}
\hline & gr/litter \\
\hline Sodium chloride $(\mathrm{NaCl})$ & 8 \\
Potassium chloride $(\mathrm{KCL})$ & 0.4 \\
Sodium bicarbonate $(\mathrm{NaHCO})$ & 0.35 \\
D-glucose (Dextrose) & 1 \\
Potassium diHydrogen phosphate $\left(\mathrm{KH}_{2} \mathrm{PO}_{4}\right)$ & 0.06 \\
Sodium EDTA (Na2-EDTA) & 0.2 \\
Sodium hydrogen phosphate, anhydrous $\left(\mathrm{Na}_{2} \mathrm{HPO}_{4}\right)$ & 0.047 \\
\hline
\end{tabular}

faced up (Fig. 1). The amnion membrane was manually stripped from the chorion layer, starting from the outer edge of the amniotic membrane and continuing towards the umbilical cord (Fig. 2). The membrane was washed several times with ice-clod PBS ( $\mathrm{pH}$ 7.2) to remove blood clots, torn pieces and cellular debris (Fig. 3).

\section{Digestion of the amniotic membrane}

Blood-free amnion was cut into pieces around $7 \mathrm{~cm}$ long and transferred with forceps to two new $50 \mathrm{ml}$ falcon conical tubes. To each tube $20 \mathrm{ml}$ pre-digestion buffer was added, and then incubated at $37{ }^{\circ} \mathrm{C}$ for 15 min with gentle shaking (30-60 RPM). Afterwards, the amnion pieces were transferred into three new $50 \mathrm{ml}$ falcon conical tubes containing $20 \mathrm{ml}$ of pre-warm $0.05 \%$ trypsin/

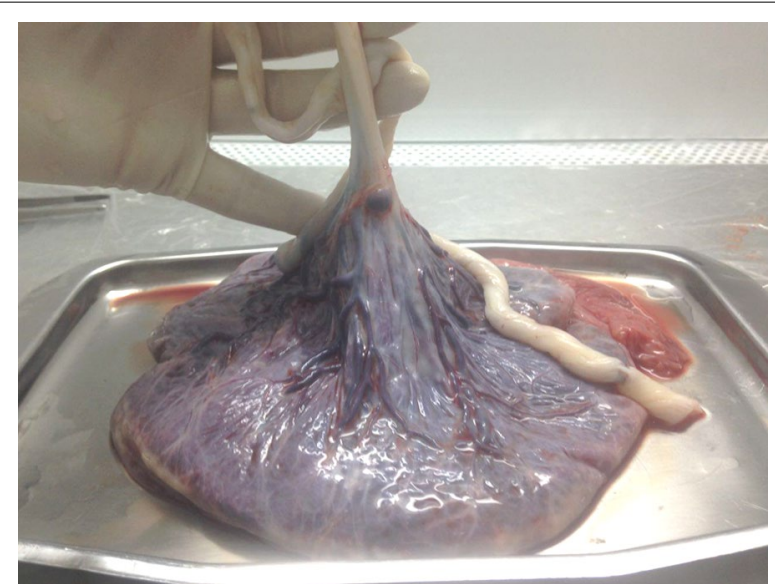

Fig. 1 The position of placing placenta in a sterile container in which the fetal surface was faced up

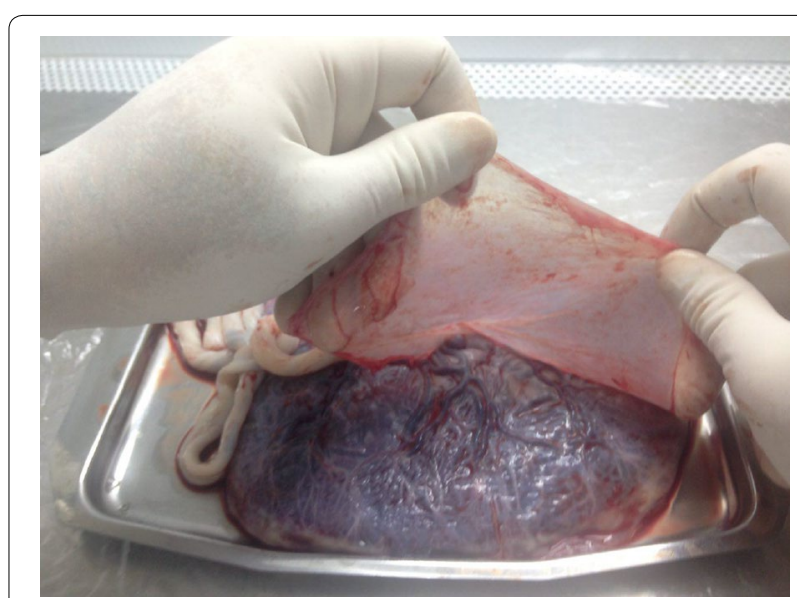

Fig. 2 Manual stripping of the amniotic membrane (upper layer) of the placenta from the chorion layer 


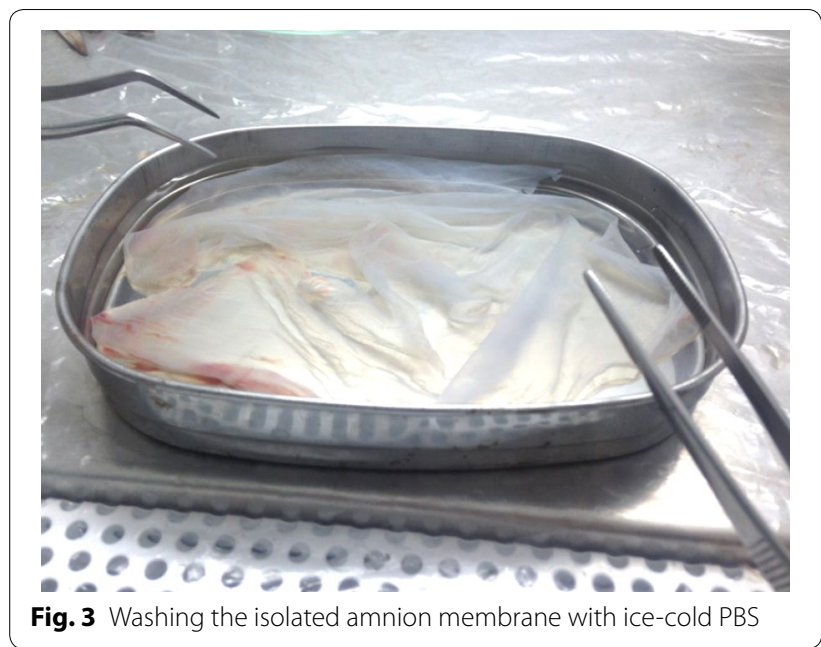

EDTA (Thermo Fisher Scientific, USA) and incubated at $37^{\circ} \mathrm{C}$ for $10 \mathrm{~min}$ with gentle shaking (first digestion). The obtained cells at this step were discarded to exclude blood clots and cellular debris and the membrane pieces were transferred into new tubes. The enzymatic digestion was followed by addition of $20 \mathrm{ml}$ pre-warm $0.05 \%$ trypsin/EDTA and incubation at $37{ }^{\circ} \mathrm{C}$ for $30 \mathrm{~min}$ with gentle shaking (second digestion). This step was repeated, then the second and the third digestions were neutralized for trypsin activity by adding $30 \mathrm{ml} \mathrm{HBSS}$, and finally centrifuged at $200 \times g$ for $5 \mathrm{~min}$ at $4{ }^{\circ} \mathrm{C}$. The cell pellets were re-suspended in $10 \mathrm{ml}$ standard culture medium, mixed together and filtered through a $100 \mu \mathrm{m}$ cell strainer (Thermo Fisher Scientific, USA). The filtrate cell number was counted using a haemocytometer and cell viability was determined using trypan blue dye exclusion.

\section{Key points}

1. It is possible that the amnion layer is peeled off from the chorion in the operating room, which has its advantages including: using less transportation medium, minimize possibility of non-sterile samples, and decreasing the amnion contamination with blood clots.

2. It is recommended that before hAECs isolation, a piece of the amniotic membrane be observed under an optical microscope $(40 \times$ magnification $)$ in order to check the status of the cells. An amniotic membrane with epithelial cells, which are notably vacuolated in their cytoplasm, is not suitable for cell isolation (Fig. 4A, B). Do not process to the next steps.

3. It is suggested that the status of the remained cells on the amniotic membrane after each digestion step be checked under an optical microscope and the membrane with highly vacuolated cells is discarded.
Checking a piece of the amniotic membrane under a microscope after each digestion step also helps to realize whether the next-step of enzymatic digestion is required or not. Accordingly, the number of required digestion steps to separate majority of the cells from the membrane may be increased or decreased.

4. It is suggested that at the end of each digestion, the membrane pieces be gently shacked using a forceps in $50 \mathrm{ml}$ tubes containing the trypsin digest to separate all the epithelial cells in case still be (loosely) attached to the membrane.

\section{hAECs immuno-phenotyping using flow cytometry}

The purity and phenotypic characteristics of freshly isolated hAECs were determined using flow cytometry. The cells $\left(4-8 \times 10^{5}\right)$ were stained with different antibodies (Table 3) or matched-isotype control IgG at $4{ }^{\circ} \mathrm{C}$ for $25 \mathrm{~min}$. Matched isotype control antibodies were used as negative controls and MSCs were employed as positive control for anti-CD90 and anti-CD105. Afterwards, the cells were washed three times using cell staining buffer (Biolegend, USA) and centrifugation at $200 \times g$ for 5 min at $4{ }^{\circ} \mathrm{C}$. Intracellular staining with FITC-conjugated-anti-cytokeratin (Biolegend, USA) was performed after fixation and cells permeabilization according to the manufacturer's instructions (eBioscience, USA). The data was acquired using a FACSCalibur system (Becton-Dickinson, CA) and analyzed using CellQuest software (Becton-Dickinson, $\mathrm{CA}$ ).

\section{hAECs proliferation assay}

Isolated hAECs were cultured in $75 \mathrm{~cm}^{2}$ tissue culture flasks at a density of $2.5 \times 10^{5}$ cells $/ \mathrm{cm}^{2}$ using standard culture medium, and then incubated at $37{ }^{\circ} \mathrm{C}$ with $5 \%$ $\mathrm{CO}_{2}$. The initial plating efficiency of the cells was determined after 2 days of incubation. The hAECs were dissociated using $0.05 \%$ trypsin-EDTA solution, whenever they were approximately $80 \%$ confluent. The cells were sub-cultured in 1:4 ratios in standard culture medium.

\section{Results}

\section{The yield and viability of $h A E C s$}

The average yield of obtained hAECs by this method was $190 \times 10^{6}$ cells with a typical range of $90-280$ million cells (Fig. 5a). Noticeably, for this yield the average obtained viability was $87 \%$ (ranging from 83 to $89 \%$ ) (Fig. 5b).

\section{Immuno-phenotyping of hAECs}

The hAECs purity achieved by this protocol was at least $95.42 \%$, as confirmed by cytokeratin analysis, an epithelial cell marker (Fig. 6a). Less than $1 \%$ of the isolated cells 

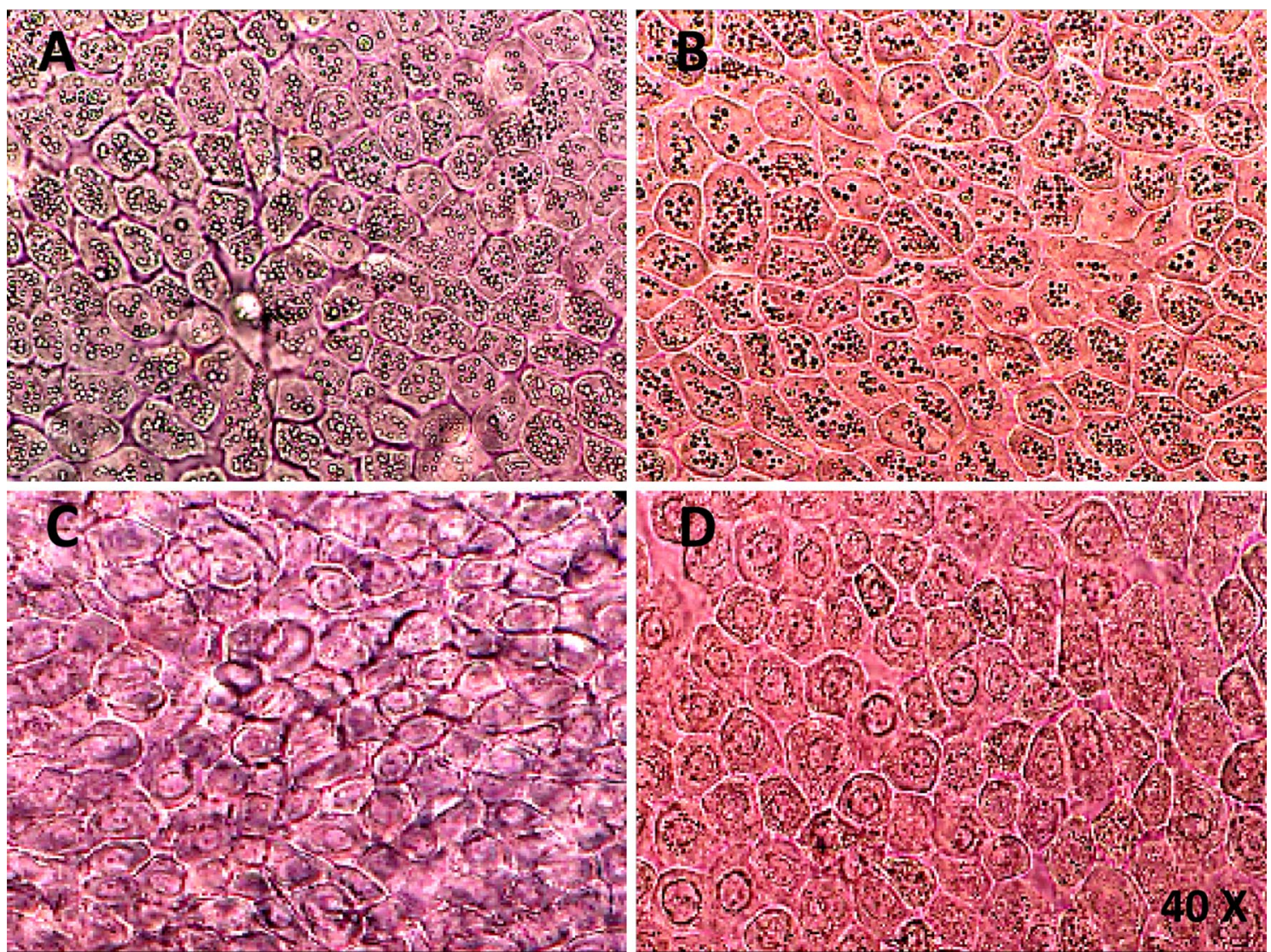

Fig. 4 Epithelial cells of the amniotic membrane under an optical microscope. A, B An amniotic membrane with epithelial cells which are fully vacuolated in their cytoplasm. C, D An amniotic membrane with normal epithelial cells which is suitable for hAECs isolation

Table 3 Used antibodies to determine phenotypic characterictics of hAECs by flow cytometry

\begin{tabular}{|c|c|c|c|}
\hline Primary antibodies/ fluorochrome & Isotype & Catalog number & Source of primary antibodies \\
\hline $\begin{array}{l}\text { Alexa Fluor }{ }^{\circledR} 488 \text { anti-Cytokeratin (pan } \\
\text { reactive) }\end{array}$ & Mouse lgG1, k (cat. no:400143) & 628608 & Biolegend, San Diego, CA, USA \\
\hline FITC anti-human CD105 & Mouse IgG1, k (cat. no:400107) & 323203 & Biolegend, San Diego, CA, USA \\
\hline FITC anti-human CD90 & Mouse IgG1, k (cat. no:400107) & 328107 & Biolegend, San Diego, CA, USA \\
\hline FITC anti-human CD45 & Mouse IgG1, k (cat. no:400107) & 368507 & Biolegend, San Diego, CA, USA \\
\hline FITC anti-human CD14 & Mouse IgG1, k (cat. no:400107) & 367115 & Biolegend, San Diego, CA, USA \\
\hline FITC anti-human CD4 & Mouse IgG1, k (cat. no:400107) & 357405 & Biolegend, San Diego, CA, USA \\
\hline FITC anti-human CD8a & Mouse IgG1, k (cat. no:400107) & 300905 & Biolegend, San Diego, CA, USA \\
\hline PE anti-human CD56 & Mouse IgG1, k (cat. no:400111) & 355503 & Biolegend, San Diego, CA, USA \\
\hline FITC anti-human CD3 & Mouse IgG1, k (cat. no:400107) & 362305 & Biolegend, San Diego, CA, USA \\
\hline FITC mouse anti-human HLA-DR & Mouse lgG ${ }_{2 a^{\prime}}$ K(cat. no: 555057) & 555560 & BD Biosciences, San Jose, CA, USA \\
\hline FITC mouse anti-human CD34 & Mouse lgG ${ }_{1}, \mathrm{k}($ cat. no: 555748) & 555821 & BD Biosciences, San Jose, CA, USA \\
\hline FITC mouse anti-human CD38 & Mouse lgG 1, k(cat. no: 555748) & 555459 & BD Biosciences, San Jose, CA, USA \\
\hline PE mouse anti-human CD44 & Mouse $\operatorname{lgG}_{1}, \mathrm{k}$ (cat. no: 550617) & 550989 & BD Biosciences, San Jose, CA, USA \\
\hline PE mouse anti-human CD9 & Mouse lgG 1, K(cat. no: 550617) & 555372 & BD Biosciences, San Jose, CA, USA \\
\hline PE mouse anti-human CD29 & Mouse lgG 1, k(cat. no: 550617) & 557332 & BD Biosciences, San Jose, CA, USA \\
\hline PE mouse anti-human CD73 & Mouse lgG 1, k(cat. no: 550617) & 550257 & BD Biosciences, San Jose, CA, USA \\
\hline Anti-human SSAE-4 PE & Mouse/ lgG3(cat. no:12-4742-42) & $12-8843-42$ & Thermo Fisher Scientific, Waltham, MA, USA \\
\hline Anti-human CD133 FITC & Mouse/lgG2b, kappa(cat. no: 11-4732-42) & $11-1339-42$ & Thermo Fisher Scientific, Waltham, MA, USA \\
\hline
\end{tabular}



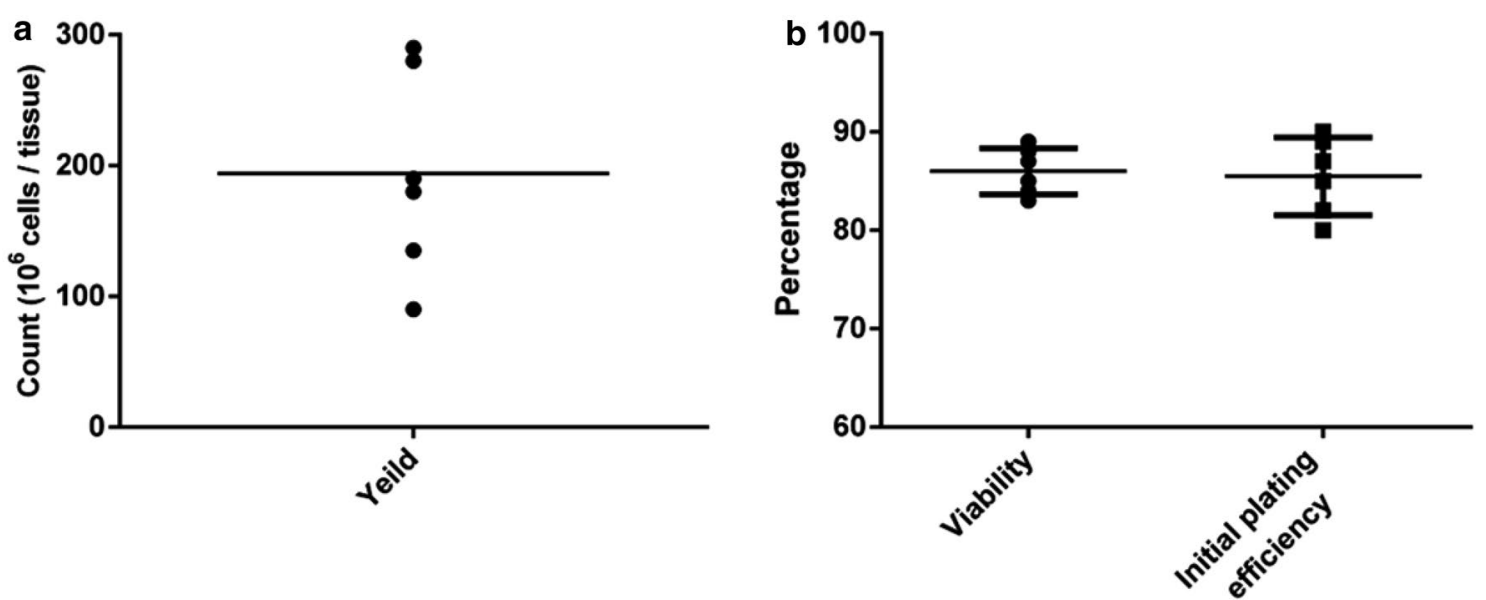

Fig. 5 Yield and viability of isolated hAECs. a The average yield of isolated hAECs was $190 \times 10^{6}$ cells with a typical range of 90-280 million cells. b An average viability obtained using this protocol was $87 \%$ (ranging from 83 to $89 \%$ ). The depicted results are representative of six independent experiments
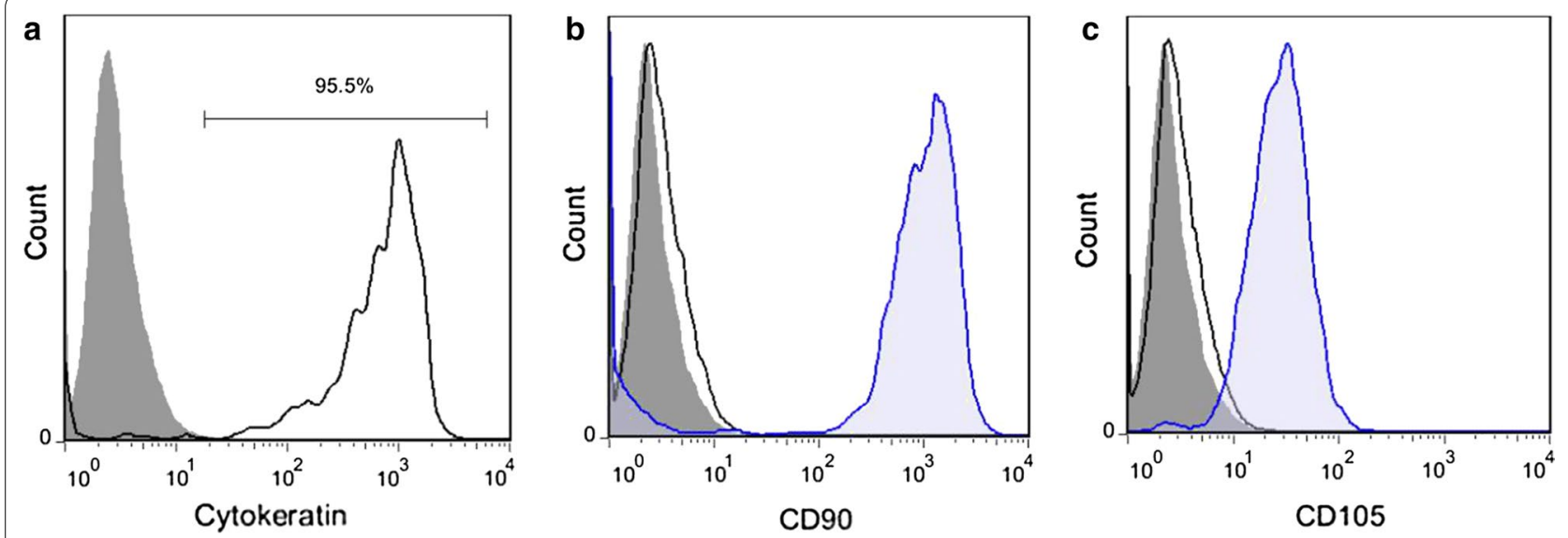

Fig. 6 The purity of isolated hAECs. a More than $95 \%$ of the isolated hAECs were positive for cytokeratin, the epithelial cell marker. b, c Less than $1 \%$ of isolated hAECs were positive for MSC markers CD90 (b) and CD105 (c). Gray shaded histogram: hAECs were stained with matched isotype control antibodies as negative controls (a-c). Blue shaded histogram: MSCs were stained with anti-CD90 (b) and anti-CD105 (c) antibodies as positive controls. Black line: hAECs were stained with anti-cytokeratin $(\mathbf{a})$, anti-CD90 (b) and anti-CD105 (c). The indicated results are representative of six independent experiments

were positive for MSC markers (CD90, CD105) (Fig. 6b, c). In addition, hAECs immuno-phenotyping from different donors showed that the isolated cells were almost a homogenous population (Table 4).

\section{The proliferation capability of hAECs}

After $48 \mathrm{~h}$, the initial plating efficiency of the isolated hAECs on culture dishes was at least $80 \%$ (Fig. 5b). Freshly isolated hAECs had the ability to proliferate for 5-6 passages in standard culture medium. In contrast with some reports $[5,16]$, hAECs which were cultured without EGF were not able to proliferate at all.

\section{Discussion}

hAECs are a type of stem cells isolated from the amniotic membrane of the placenta. In addition to stem celllike properties, which proposed hAECs as a potential candidate for regenerative medicine, it has been shown that they can be used as an immunomodulatory agent in treatment of diseases with immune pathophysiology.

hAECs isolation from the amniotic membrane is performed with enzymatic digestion of the amniotic membrane, since amnion epithelium is a single monolayer with a weak cell-cell adhesion at the lateral sides and trypsin is able to separate hAECs from the amniotic 
Table 4 Determination of markers of hAECs by flow cytometry

\begin{tabular}{|c|c|c|c|c|c|c|}
\hline Marker & Donor1 & Donor2 & Donor3 & Donor4 & Donor5 & Donor6 \\
\hline Pan cytokeratin & ++++ & ++++ & ++++ & ++++ & ++++ & ++++ \\
\hline CD73 & +++ & +++ & +++ & +++ & +++ & +++ \\
\hline SSEA-4 & +++ & +++ & +++ & +++ & +++ & +++ \\
\hline CD133 & - & - & - & - & - & - \\
\hline CD90 & - & - & - & - & - & - \\
\hline CD105 & - & - & - & - & - & - \\
\hline CD34 & - & - & - & - & - & - \\
\hline CD45 & - & - & - & - & - & - \\
\hline CD9 & ++ & - & + & + & - & + \\
\hline CD38 & - & - & - & - & - & - \\
\hline HLA-DR & - & - & - & - & - & - \\
\hline Integrin- $\beta 2$ (CD29) & + & + & + & + & ++ & + \\
\hline Hyaluronic acid receptor (CD44) & - & + & + & + & ++ & + \\
\hline CD56 & $-/+$ & $-/+$ & $-/+$ & $-l+$ & $-/+$ & $-l+$ \\
\hline CD14 & + & ++ & - & + & ++ & - \\
\hline CD3 & - & - & - & - & - & - \\
\hline CD4 & - & - & - & - & - & - \\
\hline CD8 & - & - & - & - & - & - \\
\hline
\end{tabular}

- , Not determined; $\mp$, very low expression $(<10 \%) ;+$, low expression $(10-30 \%) ;++$, intermediate expression $(30-60 \%) ;+++$, high expression $(60-90 \%) ;++++$, very high expression (>90\%)

basement membrane [11]. There is a notable variability in the purity, yield and viability of hAECs isolation using previous protocols $[3,10-15]$. These variations might be related to several factors including mother related parameters (e.g. age, gestational week and delivery type), size and quality of the placenta (which is affected by duration of the time between the delivery and hAECs isolation), transportation condition (such as cold chain), residual blood on the tissue (in spite of extensive washing), type and concentration of the enzyme solution, and enzymatic digestion time [3, 10-14]. A disadvantage of these methods for cell isolation is that achieving a desirable yield come at the expense of losing the cell viability and purity. The presented protocol at this study resulted in hAECs isolation with high yield (to our knowledge, the highest yield reported yet), viability and purity.

Using the proposed modified protocol, an average yield of $190 \times 10^{6}$ hAECs (in range of 90-280 million cells) was achieved compared to the average yield of $8 \times 10^{6}-$ $120 \times 10^{6}$ cells obtained using previous protocols [3, $10-12,14,15]$. Regarding the fact that a consistent protocol was followed for hAECs isolation from all donors in the current study, the observed extensive variability in cell yield might be related to quality of the placenta obtained from different donors. The mean viability of isolated hAECs in this study was $87 \%$, compared to previously reports with a range from 83 to $99 \%$ [14]. Previous methods reported that high yield of the viable cells was accompanied contamination with other cell populations $[10,11,14]$. However, the current study reported a highly viable hAECs at high yield and minimum contamination with MSCs. These findings could be mainly attributed to two factors, a shorter incubation time compared to previous protocols and discarding the first digestion. Regarding the facts that a part of blood clots and cellular debris are not removed after extensive washing of the amniotic membrane with PBS, consequently these parts are separated from the membrane in the first step of digestion.

The initial plating efficiency of the cells in the current study was at least $80 \%$ after $48 \mathrm{~h}$, moreover the cells continued to proliferate for 5-6 passages in standard culture medium. It is found that in addition to the initial viability of the isolated cells which is an essential factor for determining long-term survival of hAECs and their proliferation, the initial plating efficiency is critical as well.

According to the results, isolated hAECs from different placentas were highly homogeneous based on the expression of epithelial (cytokeratin), MSC (CD105, CD73, CD90), embryonic stem cell (SSEA-4), hematopoietic stem cell (CD34), and immunologic (HLA-DR, CD56, CD3, CD4, CD8) markers and adhesion molecules (CD29). However, there were differences in the level of CD9, CD14 and CD44 between different donors.

In summary, using the new modified protocol a high hAECs yield with high viability and purity was obtained, and the cells kept their proliferation ability until passage 5-6. 


\section{Limitation}

A limitation of this technique is that increasing the cells yield may result in losing the viability, purity and initial plating efficiency.

\begin{abstract}
Abbreviations
DMEM: Dulbecco's modified eagle's medium; RPMI: Roswell park memorial institute; PBS: phosphate-buffered saline; FBS: fetal bovine serum; EGF: epidermal growth factor; hAECs: human amniotic epithelial cells; HBSS: Hanks' balanced salt solution; HBV: hepatitis B virus; HCV: hepatitis C virus; HIV: human immunodeficiency virus.
\end{abstract}

\section{Authors' contributions}

HM, SG carried out the experiments and preformed statistical analysis. NE participated in the design of the experiments and obtained funding for the work. NT participated in the study design. FK, BK provided the placenta tissue and participated in the design of the experiments. AR drafted the manuscript and participated in the study design. All authors read and approved the final manuscript.

\section{Author details}

${ }^{1}$ Department of Immunology, Faculty of Medicine, Isfahan University of Medical Sciences, Hezar-Jereeb Ave, Isfahan, Iran. ${ }^{2}$ Immunology Research Center (IRC), Iran University of Medical Sciences, Tehran, Iran. ${ }^{3}$ Department of Laboratory Medicine, Kashan University of Medical Sciences, Kashan, Iran. ${ }^{4}$ Department of Gynecology and Obstetrics, Al-Zahra Hospital, Isfahan University of Medical Sciences, Isfahan, Iran.

\section{Acknowledgements}

The authors thank all individuals who participated in this study.

\section{Competing interests}

The authors declare that they have no competing interests.

\section{Availability of data and materials}

All data generated or analyzed during this study are included in this published article.

\section{Consent for publication}

Not applicable.

\section{Ethics approval and consent to participate}

This study was approved by the Ethics Committee of Isfahan University of Medical Science. All contributors signed informed consent before entering the study.

\section{Funding}

This study was financially supported by Kashan University of Medical Sciences (KAUMS).

\section{Publisher's Note}

Springer Nature remains neutral with regard to jurisdictional claims in published maps and institutional affiliations.
Received: 9 June 2017 Accepted: 26 October 2017

Published online: 02 November 2017

\section{References}

1. Miki T, Lehmann T, Cai H, Stolz DB, Strom SC. Stem cell characteristics of amniotic epithelial cells. Stem cells. 2005;23:1549-59.

2. van Herendael B, Oberti C, Brosens I. Microanatomy of the human amniotic membranes: a light microscopic, transmission, and scanning electron microscopic study. Am J Obstet Gynecol. 1978;131:872-80.

3. Miki T, Marongiu F, Dorko K, Ellis E, Strom SC. Isolation of amniotic epithelial stem cells. Curr Protoc Stem Cell Biol. 2010. https://doi. org/10.1002/9780470151808.sc01e03s12.

4. Kakishita K, Elwan MA, Nakao N, Itakura T, Sakuragawa N. Human amniotic epithelial cells produce dopamine and survive after implantation into the striatum of a rat model of Parkinson's disease: a potential source of donor for transplantation therapy. Exp Neurol. 2000;165:27-34.

5. Liu YH, Vaghjiani V, Tee JY, To K, Cui P, Oh DY, et al. Amniotic epithelial cells from the human placenta potently suppress a mouse model of multiple sclerosis. PLoS ONE. 2012;7:e35758.

6. McDonald CA, Payne NL, Sun G, Moussa L, Siatskas C, Lim R, et al. Immunosuppressive potential of human amnion epithelial cells in the treatment of experimental autoimmune encephalomyelitis. J Neuroinflamm. 2015;12:112.

7. Ilancheran S, Michalska A, Peh G, Wallace EM, Pera M, Manuelpillai U. Stem cells derived from human fetal membranes display multilineage differentiation potential. Biol Reprod. 2007;77:577-88.

8. Niknejad H, Peirovi H, Jorjani M, Ahmadiani A, Ghanavi J, Seifalian AM. Properties of the amniotic membrane for potential use in tissue engineering. Eur Cells Mater. 2008;15:88-99.

9. Akle C, Welsh K, Adinolfi M, Leibowitz S, McColl I. Immunogenicity of human amniotic epithelial cells after transplantation into volunteers. Lancet. 1981;318:1003-5.

10. Gomez Dominguez R. Human amniotic epithelial cells: isolation and characterisation. Giessen: Laufersweiler Publishers; 2008.

11. Gramignoli R, Srinivasan RC, Kannisto K, Strom SC. Isolation of human amnion epithelial cells according to current good manufacturing procedures. Curr Protoc Stem Cell Biol. 2016. https://doi.org/10.1002/cpsc.2.

12. Murphy S, Rosli S, Acharya R, Mathias L, Lim R, Wallace E, et al. Amnion epithelial cell isolation and characterization for clinical use. Curr Protoc Stem Cell Biol. 2010. https://doi.org/10.1002/9780470151808.sc01e06s13.

13. Tabatabaei M, Mosaffa N, Nikoo S, Bozorgmehr M, Ghods R, Kazemnejad $S$, et al. Isolation and partial characterization of human amniotic epithelial cells: the effect of trypsin. Avicenna J Med Biotechnol. 2014;6:10.

14. Murphy SV, Kidyoor A, Reid T, Atala A, Wallace EM, Lim R. Isolation, cryopreservation and culture of human amnion epithelial cells for clinical applications. J Vis Exp. 2014. https://doi.org/10.3791/52085.

15. Toda A, Okabe M, Yoshida T, Nikaido T. The potential of amniotic membrane/amnion-derived cells for regeneration of various tissues. J Pharmacol Sci. 2007;105:215-28.

16. Chen YT, Li W, Hayashida Y, He H, Chen SY, Tseng DY, et al. Human amniotic epithelial cells as novel feeder layers for promoting ex vivo expansion of limbal epithelial progenitor cells. Stem Cells. 2007;25:1995-2005. 